\title{
Guest Editorial for the Special Issue on FORmal methods for the quantitative Evaluation of Collective Adaptive SysTems (FORECAST)
}

\author{
MAURICE H. TER BEEK, Consiglio Nazionale delle Ricerche (CNR), Istituto di Scienza e Tecnologie \\ dell'Informazione (ISTI) \\ MICHELE LORETI, Università di Camerino, Scuola di Scienze e Tecnologie
}

CCS Concepts: • Computing methodologies $\rightarrow$ Model development and analysis; • Theory of computation $\rightarrow$ Models of computation; Semantics and reasoning;

Additional Key Words and Phrases: Formal methods, Collective Adaptive Systems, system specification and verification

\section{ACM Reference format:}

Maurice H. ter Beek and Michele Loreti. 2018. Guest Editorial for the Special Issue on FORmal methods for the quantitative Evaluation of Collective Adaptive SysTems (FORECAST). ACM Trans. Model. Comput. Simul. 28, 2, Article 8 (February 2018), 4 pages.

https://doi.org/10.1145/3177772

Collective Adaptive Systems (CAS) consist of a large number of spatially distributed heterogeneous entities with decentralized control and varying degrees of complex autonomous behavior that may be competing for shared resources even when collaborating to reach common goals. It is important to carry out thorough quantitative modeling and analysis and verification of their design to investigate all aspects of their behavior before they are put into operation. This requires combinations of formal methods (e.g., stochastic process algebras and associated verification techniques, such as quantitative model checking) and applied mathematics (e.g., mean field/continuous approximation and control theory) which, moreover, scale to large-scale CAS.

In this context, the FORECAST workshop on FORmal methods for the quantitative Evaluation of Collective Adaptive SysTems-held in Vienna (Austria) on July 8, 2016 as a satellite event of the 4th federated event on Software Technologies: Applications and Foundations (STAF 2016)-was organized to raise awareness in the software engineering and formal methods communities of the particularities of CAS, and the design and control problems that they bring. The guest editors of this special issue served as co-chairs of the workshop's Program Committee and were responsible for its proceedings [4]. FORECAST was sponsored by the FP7-ICT-600708 European project QUANTICOL (A Quantitative Approach to Management and Design of Collective and Adaptive Behaviours), that ran from 2013 to 2017 [3, 5, 7]. We thank the project's participants and in particular its coordinator, Jane Hillston, for entrusting us with the workshop's organization.

Authors' addresses: M. H. ter Beek, Consiglio Nazionale delle Ricerche (CNR), Istituto di Scienza e dell'Informazione (ISTI), Via G. Moruzzi 1, Pisa (PI), 56125, Italy; email: maurice.terbeek@isti.cnr.it; M. Loreti, Università di Camerino, Scuola di Scienze e Tecnologie, Via Madonna delle Carceri 9, Camerino (MC), 62032, Italy; email: michele.loreti@unicam.it.

Permission to make digital or hard copies of part or all of this work for personal or classroom use is granted without fee provided that copies are not made or distributed for profit or commercial advantage and that copies bear this notice and the full citation on the first page. Copyrights for third-party components of this work must be honored. For all other uses, contact the Owner/Author.

2018 Copyright is held by the owner/author(s).

1049-3301/2018/02-ART8

https://doi.org/10.1145/3177772 
Following this highly successful workshop, an open call was announced for the submission of papers either related to the papers presented at FORECAST or unpublished high-quality papers related to the themes of FORECAST on the crossroads of formal methods, applied mathematics, and software engineering. As a result, six papers were submitted, equally distributed over the two types of submissions sought for, all of which were peer reviewed by three reviewers. One paper was rejected, while all others underwent two rounds of reviews. We have three papers that have undergone the Replicating Computational Results (RCR) review process [1, 6, 10]. Their respective $\mathrm{RCR}$ reports are included in this special issue $[2,8,11]$.

The article by Aldini [1] proposes a process-algebraic framework for modeling and verifying trusted CAS. To favor reuse, the system and trust models can be specified separately, only to be integrated at the semantic level. Through a combination of behavioral equivalence checking and model checking against branching-time temporal logic with trust predicates, the framework allows comparative analyses of different trust models and analyses of the effects of attacks to the trust models. The applicability of the formal framework is illustrated by means of two representative use cases: the security analysis of a trust-incentive service management system and a comparison of two different reputation systems. The RCR review by Maurice ter Beek [2] affirms that it was straightforward to replicate all (model-checking) experiments described in the article in reasonable time using a standard laptop.

The article by Galpin et al. [6] uses CARMA (Collective Adaptive Resource-sharing Markovian Agents), a specification language recently introduced for modeling CAS, to model spatially distributed systems in which the desired model lies between an individual-based (microscopic) and a population-based (macroscopic) spatial model. The impact on the system dynamics of changes to the topology of paths is studied via simulation. The provided experiments show that it is difficult to predict the effect of changes to the network structure and that even small variations can produce significant effects. The RCR review by Michele Loreti [8] concluded that the experiments could be correctly replicated and the obtained results are compatible with the ones presented in [6].

The article by Marin et al. [9] presents a novel resource allocation scheme for bandwidth assignment in wireless sensor networks (WSNs), called Fair Allocation Control Window (FACW), together with a product-form queuing network model of the protocol capable of predicting the network model's performance in polynomial time by means of a convolution algorithm. Hence, it can be used to tackle the problems of the state space explosion typical of CAS. The proposed model is studied with several what-if analyses, each focusing on different system parameters. The model solutions are validated against performance indices obtained with discrete event simulation of the FACW protocol in WSNs, to which it compares favorably.

The article by Sebastio et al. [10] proposes a novel holistic approach to task scheduling in volunteer clouds. Volunteer clouds are decentralized cloud systems based on collaborative task execution, in which clients voluntarily share their spare computational resources to overcome the scalability limitations of cloud computing. By using simulation-based statistical analysis techniques-in particular, statistical model checking-the authors show that in the case of computation-intensive workloads, their approach outperforms existing distributed task scheduling algorithms in terms of perceived quality of service owing to its collective, decentralized, collaborative, and self-adaptive nature. The analysis considered a realistic workload benchmark provided by Google. The RCR review by Andrea Vandin [11] focuses on the prototypical tool implementation used in the article to perform such analysis and it states that the software was straightforward to install and use, and a representative part of the experimental results from the article could be reproduced in reasonable time using a standard laptop machine. 
The article by Viroli et al. [12] presents an approach to aggregate computing based on field calculus (and associated toolchain) as computational model. Aggregate computing supports the design of complex and large-scale software systems by focusing on the global behavior of a collection of devices, thus abstracting away from their individual behavior. The authors propose a methodology to engineer resilient CAS by specifying self-stabilizing systems in a spatial-temporal domain using a macro-programming language. With the aim of providing reusable blocks of distributed behavior that are correct and smoothly composable, they study the possibility of substituting blocks by functionally equivalent ones that are more efficient in terms of performance or have more desirable dynamics without affecting the overall system behavior and resilience, i.e., guaranteed to eventually attain a correct and stable final state despite any transient perturbation in state or topology.

Preparations for this special issue began soon after the FORECAST workshop took place; it has taken roughly one and a half years for the articles to be ready for publication. A rigorous peerreview process has ensured that the selected papers represent cutting-edge research on formal methods for the quantitative evaluation of CAS. This would not have been possible without the contributions of numerous reviewers, whom we would like to thank for preparing detailed referee reports and respecting the timelines of this special issue. We also thank all contributing authors for their hard work in presenting their research and for revising their articles in time according to the reviewers' comments. Finally, we thank the editorial assistant for ACM TOMACS, Holley Cornetto, for her management, and the journal's editor-in-chief, Adelinde Uhrmacher, for having agreed to publish this special issue and for her encouragement and support throughout the process.

\section{REFERENCES}

[1] Alessandro Aldini. 2018. Design and verification of trusted collective adaptive systems. Transactions on Modeling and Computer Simulation 28, 2, Article 9, 27 pages.

[2] Maurice H. ter Beek. 2018. Replicated computations results (RCR) report for "design and verification of trusted collective adaptive systems." Transactions on Modeling and Computer Simulation 28, 2, Article 10, 3 pages.

[3] Maurice H. ter Beek, Luca Bortolussi, Vincenzo Ciancia, Stefania Gnesi, Jane Hillston, Diego Latella, and Mieke Massink. 2014. A quantitative approach to the design and analysis of collective adaptive systems for smart cities. ERCIM News98. Retrieved January 30, 2018 from http://ercim-news.ercim.eu/en98/special/ a-quantitative-approach-to-the-design-and-analysis-of-collective-adaptive-systems-for-smart-cities

[4] Maurice H. ter Beek and Michele Loreti (eds.). 2016. Proceedings of the Workshop on FORmal methods for the quantitative Evaluation of Collective Adaptive SysTems (FORECAST'16). Electronic Proceedings in Theoretical Computer Science, Vol. 217. DOI : http://dx.doi.org/10.4204/EPTCS.217 arXiv:1607.02001v1

[5] Marco Bernardo, Rocco De Nicola, and Jane Hillston (eds.). 2016. Formal Methods for the Quantitative Evaluation of Collective Adaptive Systems: Advanced Lectures of the 16th International School on Formal Methods for the Design of Computer, Communication, and Software Systems (SFM'16). Lecture Notes in Computer Science, Vol. 9700. Springer, Berlin. DOI : http://dx.doi.org/10.1007/978-3-319-34096-8

[6] Vashti Galpin, Natalia Zoń, Pia Wilsdorf, and Stephen Gilmore. 2018. Mesoscopic modelling of pedestrian movement using CARMA and its tools. Transactions on Modeling and Computer Simulation 28, 2, Article 11, 26 pages.

[7] Jane Hillston, Jeremy Pitt, Martin Wirsing, and Franco Zambonelli. 2015. Collective adaptive systems: qualitative and quantitative modelling and analysis (Dagstuhl seminar 14512). Dagstuhl Reports 4, 12, 68-113. DOI : http://dx.doi.org/ 10.4230/DagRep.4.12.68

[8] Michele Loreti. 2018. Replicated computations results (RCR) report for "mesoscopic modelling of pedestrian movement using CARMA and its tools." Transactions on Modeling and Computer Simulation 28, 2, Article 12, 3 pages.

[9] Andrea Marin, Sabina Rossi, Dario Burato, Andrea Sina, and Matteo Sottana. 2018. A product-form model for the performance evaluation of a bandwidth allocation strategy in WSNs. Transactions on Modeling and Computer Simulation 28, 2, Article 13, 23 pages.

[10] Stefano Sebastio, Michele Amoretti, Alberto Lluch Lafuente, and Antonio Scala. 2018. A holistic approach for collaborative workload execution in volunteer clouds. Transactions on Modeling and Computer Simulation 28, 2, Article 14, 27 pages. 
[11] Andrea Vandin. 2018. Replicated computations results (RCR) report for "a holistic approach for collaborative workload execution in volunteer clouds." 28, 2, Article 15, 3 pages.

[12] Mirko Viroli, Giorgio Audrito, Jacob Beal, Ferruccio Damiani, and Danilo Pianini. 2018. Engineering resilient collective adaptive systems by self-stabilisation. Transactions on Modeling and Computer Simulation 28, 2, Article 16, 28 pages.

Received December 2017; accepted December 2017 